\title{
Outrunning COVID-19: a hypothesis that promoting aerobic exercise could prevent severe disease
}

\author{
Chris Kenyon ${ }^{1}$ \\ ${ }^{1}$ Institute of Tropical Medicine
}

May 6, 2020

\begin{abstract}
NA

Dear Editors,

Sriram et al., recently argued that an imbalance between angiotensin converting enzyme-1 (ACE1) and ACE2 explained why only a small proportion of those infected with SARS-CoV-2 develop severe COVID-19 disease (Sriram \& Insel, 2020). Their theory provides a parsimonious explanation for why all around the world severe COVID-19 is closely linked to older ages as well as certain comorbidities such as hypertension, diabetes, obesity and heart failure but not others such as asthma, COPD and HIV (Sriram \& Insel, 2020). Older age as well as hypertension, diabetes, obesity and heart failure are all associated with a relative upregulation of the pro-inflammatory ACE1/angiotensin II/angiotensin 2 receptor 1-axis and downregulation of the anti-inflammatory ACE2/angiotensin (1-7)/Mas axis. This imbalance is aggravated by SARS-CoV2 which uses ACE2 for cell entry and results in downregulation of ACE2 expression in alveolar cells and elsewhere. Individuals with this ACE1 proinflammatory, pro-apoptotic, pro-fibrotic bias are more likely to respond to COVID-19 with an untargeted, hyperinflammatory response that can progress to an acute respiratory distress syndrome-type illness before their adaptive immune system can adequately respond to the viral infection (Sriram \& Insel, 2020). Individuals with a balanced ACE1/ACE2 system by contrast do not develop this hyperinflammatory response and are able to mount an effective immune response and eradicate the virus with few or no symptoms.
\end{abstract}

Whilst this is likely a simplification of the pathophysiology of severe COVID-19, it does potentially offer a cheap, low risk strategy for whole populations to reduce their risk of severe COVID-19 - exercise. A number of studies in a range of animals, including humans, have found that exercise results in a number of health and metabolic benefits including upregulating ACE2 and downregulating ACE1 (Echeverria-Rodriguez, GallardoOrtiz, Del Valle-Mondragon, \& Villalobos-Molina, 2020; Magalhaes et al., 2020). One study for example estimated that every hour run prolongs one's life by 7 hours (Lee et al., 2017). These considerations led us to add to the hypothesis of Sriram et al., that the promotion of exercise, particularly in those at risk for severe COVID-19 will reduce the probability of them developing severe COVID-19. The available evidence is that both moderate- and high intensity aerobic exercise result in the rapid upregulation of the ACE2 versus ACE1 axis (Magalhaes et al., 2020). Randomized controlled trials in animal models of COVID-19 (Chan et al., 2020) or high-risk groups of humans would be required to test if this is protective against severe COVID-19 as well as to ascertain what the optimal training regimens for specific risk profiles would be. Pending these studies, a reasonable argument could made that the health benefits of exercise in these risk groups are so well established that this novel hypothesis could still be shared with target groups (Lee et al., 2017).

\section{Authors' contributions}


CK conceptualized the article and wrote it up as a manuscript.

\section{Funding}

Nil

\section{Conflict of interest}

The author declares that he/she has no competing interests.

\section{Ethical approval}

No ethics approval was necessary.

\section{Informed consent}

Not applicable

\section{Acknowledgements}

Nil

\section{References}

Chan, J. F., Zhang, A. J., Yuan, S., Poon, V. K., Chan, C. C., Lee, A. C., . . . Yuen, K. Y. (2020). Simulation of the clinical and pathological manifestations of Coronavirus Disease 2019 (COVID-19) in golden Syrian hamster model: implications for disease pathogenesis and transmissibility. Clin Infect Dis . doi:10.1093/cid/ciaa325

Echeverria-Rodriguez, O., Gallardo-Ortiz, I. A., Del Valle-Mondragon, L., \& Villalobos-Molina, R. (2020). Angiotensin-(1-7) Participates in Enhanced Skeletal Muscle Insulin Sensitivity After a Bout of Exercise. $J$ Endocr Soc, 4 (2), bvaa007. doi:10.1210/jendso/bvaa007

Lee, D.-c., Brellenthin, A. G., Thompson, P. D., Sui, X., Lee, I.-M., \& Lavie, C. J. (2017). Running as a key lifestyle medicine for longevity.Progress in cardiovascular diseases, 60 (1), 45-55.

Magalhaes, D. M., Nunes-Silva, A., Rocha, G. C., Vaz, L. N., de Faria, M. H. S., Vieira, E. L. M., . . . Simoes, E. S. A. C. (2020). Two protocols of aerobic exercise modulate the counter-regulatory axis of the renin-angiotensin system. Heliyon, 6 (1), e03208. doi:10.1016/j.heliyon.2020.e03208

Sriram, K., \& Insel, P. A. (2020). A hypothesis for pathobiology and treatment of COVID-19: the centrality of ACE1/ACE2 imbalance. Br J Pharmacol . doi:10.1111/bph.15082 